\title{
The Effect of Knudsen Number on Transient Times During Chemical Vapor Deposition
}

\begin{abstract}
Matthias K. Gobbert ${ }^{1}$ and Timothy S. Cale ${ }^{2}$
Models for the individual steps used to fabricate integrated circuits (ICs) are of interest in order to improve fabrication efficiency and improve process designs. Here we focus on deposition from the gas stream, in which the dominant species is an inert carrier gas, as it flows across a wafer on which ICs are being fabricated. We model the transport of gaseous species to the surface and heterogeneous (surface) chemical reactions for chemical vapor deposition using a kinetic transport and reaction model (KTRM), which is represented by a system of linear Boltzmann equations. The model is valid for a range of pressures and for length scales from nanometers to decimeters, making it suitable for multiscale models. We present transient simulation results for transport of reactants into an inherently three-dimensional prototypical micron scale trench/via structure for a wide range of Knudsen numbers. The results highlight the capabilities of the KTRM and its implementation, and demonstrate that the transients last longer for lower Knudsen numbers than for higher Knudsen numbers. We briefly discuss how the KTRM might be used in a multiscale computational model.
\end{abstract}

\section{INTRODUCTION}

Several important manufacturing processes for integrated circuits (ICs) involve the flow of gaseous reactants over the wafer(s) on which the ICs are being made. Each process can occur at low (0.01 Torr), moderate, or high (atmospheric) pressures. Correspondingly, the average distance that a molecule travels before colliding with another molecule (the mean free path $\lambda$ ) ranges from less than 0.1 micron (micrometer) to over $1 \mathrm{~cm}$. On the one hand, the size of the structures created on the wafer during IC fabrication (called features) is now well below 1 micron. On the other hand, the size of the chemical reactor through which the gas flow takes place is on the order of decimeters. The appropriate transport model at a given combination of pressure and length scale is determined by the Knudsen number Kn, defined as the ratio of the mean free path to the length scale of interest $\mathrm{Kn}:=\lambda / L$. The Knudsen number arises as the relevant dimensionless group in kinetic equations [1], and serves as a guide to the type of model needed: (i) For small values Kn $<0.01$,

\footnotetext{
${ }^{1}$ Department of Mathematics and Statistics, University of Maryland, Baltimore County, 1000 Hilltop Circle, Baltimore, MD 21250 , U.S.A.

${ }^{2}$ Focus Center - New York, Rensselaer: Interconnections for Hyperintegration, Isermann Department of Chemical and Biological Engineering, Rensselaer Polytechnic Institute, CII 6015, 110 8th Street, Troy, NY 12180-3590, U.S.A.
} 
the usual continuum models describe the gas flow well. (ii) At intermediate values $\mathrm{Kn} \approx 1$, kinetic models based on the Boltzmann transport equation capture the influence of both transport of and collisions among the molecules; this is called the transition regime. (iii) For large values $\mathrm{Kn}>100$, kinetic models are still appropriate, with the collision term being small; this is called the free molecular flow, or ballistic transport, regime. We are interested in models for species transport and chemical reactions on the sub-micron scale ( $1 \mathrm{~nm}$ to 10 micron) to millimeter scale (10 micron to 1000 micron), over a range of pressures. This results in Knudsen numbers ranging from less than 0.01 to greater than 100; i.e., all three transport regimes.

The focus of this paper is to study the effect of Kn on the time it takes for transients to be essentially complete; e.g., how long does it take for the effects of a change in fluxes into the modeled domain to stop changing the local concentration and kinetic density through the domain. The studies presented here extend reports in $[2,3,4]$. Studies in Ref. [2] focus on the parallel scalability of the numerical method on a distributed-memory cluster. Ref. [3] focuses on a full introduction to the modeling and careful reference simulations that provide a validation of the model, and Ref. [4] focuses on studies of the numerical method and its convergence that provide a validation of the numerical method. The studies in Ref. [3] validated the model against EVOLVE (discussed in [5] and references therein), a well-established code that provides steady state solutions to low pressure transport and reaction inside features on patterned wafers. The KTRM computed distribution of flux of incoming species along the surface of a feature compares well with that computed by EVOLVE. The agreement improves with increasing number of velocity terms, as expected, as confirmed by the studies focussing on the numerical method in Ref. [4]. The studies in $[2,3,4]$ considered the Knudsen number as a dimensionless group that characterizes the flow regime, but did not specifically address the Kn dependence on how long it takes for a transient to disappear. Since the transient time dependency needs to be taken into account when developing an efficient multiscale simulator, we extend our work to include this issue here.

These authors have coupled models on several length scales, from feature scale to reactor scale, to form a single, or concurrent multiscale reactor simulator using a pseudosteady state approach $[6,7]$. Continuum models were used for all but the feature scale; free molecular flow was appropriate at that scale, as Kn > 100 . The current work provides the basis for creating a multiscale model which is valid over a wider pressure range, uses finite element methods and parallel code on all scales, and can deal with process transients; e.g., in atomic layer deposition $[8,9,10,11,12]$. Such a multiscale model will require well-tested and validated models and numerical methods for each length scale of interest [5].

The following section summarizes the kinetic transport and reaction model (KTRM) developed for the processes under consideration. Section 3 briefly describes the numerical methods used. The main part of this paper is the presentation and discussion of simulation results for a micron scale model of chemical vapor 
deposition (CVD) in Section 4. Finally, Section 5 summarizes the conclusions drawn from the numerical results.

\section{THE MODEL}

We have developed the kinetic transport and reaction model (KTRM) [3, 9, 10] to model flow of reactive species in a gas flow dominated by an inert carrier that is assumed to be an order denser than the reactive species. The KTRM is then represented by a system of linear Boltzmann equations, one for each of the $n_{s}$ reactive species

$$
\frac{\partial f^{(i)}}{\partial t}+\mathbf{v} \cdot \nabla_{\mathbf{x}} f^{(i)}=\frac{1}{\mathrm{Kn}} Q_{i}\left(f^{(i)}\right), \quad i=1, \ldots, n_{s},
$$

with the linear collision operators

$$
Q_{i}\left(f^{(i)}\right)(\mathbf{x}, \mathbf{v}, t)=\int_{\mathbb{R}^{3}} \sigma_{i}\left(\mathbf{v}, \mathbf{v}^{\prime}\right)\left[M_{i}(\mathbf{v}) f^{(i)}\left(\mathbf{x}, \mathbf{v}^{\prime}, t\right)-M_{i}\left(\mathbf{v}^{\prime}\right) f^{(i)}(\mathbf{x}, \mathbf{v}, t)\right] d \mathbf{v}^{\prime}
$$

where $\sigma_{i}\left(\mathbf{v}, \mathbf{v}^{\prime}\right)=\sigma_{i}\left(\mathbf{v}^{\prime}, \mathbf{v}\right) \geq 0$ is a given collision frequency model and $M_{i}(\mathbf{v})$ denotes the Maxwellian distribution of species $i$. See [3] for a derivation of the model and more details on its assumptions and non-dimensionalization. The left-hand side of (2.1) models the advective transport of molecules of species $i$ (local coupling of spatial variations via the gradient $\nabla_{\mathbf{x}} f^{(i)}$ ), while the right-hand side models the effect of collisions (global coupling of all velocities in the integral operators $Q_{i}$ ). The Knudsen number arises as the relevant dimensionless group in (2.1), because the transport on the left-hand side is non-dimensionalized with respect to the typical domain size, while the collision operator on the right-hand side is non-dimensionalized with respect to the mean free path. Thus, the values of Kn are affected by both the scale of interest of the model and by the operating conditions of the chemical reactor. The unknown functions $f^{(i)}(\mathbf{x}, \mathbf{v}, t)$ in this kinetic model represent the (scaled) probability density, called the kinetic density in the following, that a molecule of species $i=1, \ldots, n_{s}$ at position $\mathbf{x} \in \Omega \subset \mathbb{R}^{3}$ has velocity $\mathbf{v} \in \mathbb{R}^{3}$ at time $t$. Its values need to be determined at all points $\mathbf{x}$ in the three-dimensional spatial domain $\Omega$ and for all three-dimensional velocity vectors $\mathbf{v}$ at all times $0<t \leq t_{\text {fin }}$. This high dimensionality of the space of independent variables is responsible for the numerical complexity of kinetic models, as six dimensions need to be discretized, at every time step for transient simulations. Notice that while the equations in (2.1) appear decoupled, they actually remain coupled through the boundary condition at the wafer surface that models the surface reactions and is of crucial importance for the applications under consideration. 


\section{THE NUMERICAL METHOD}

The numerical method for (2.1) needs to discretize the spatial domain $\Omega \subset \mathbb{R}^{3}$ and the (unbounded) velocity space $\mathbb{R}^{3}$. We start by approximating each $f^{(i)}(\mathbf{x}, \mathbf{v}, t)$ by an expansion $f_{K}^{(i)}(\mathbf{x}, \mathbf{v}, t):=\sum_{\ell=0}^{K-1} f_{\ell}^{(i)}(\mathbf{x}, t) \varphi_{\ell}(\mathbf{v})$. Here, the basis functions $\varphi_{\ell}(\mathbf{v})$ in velocity space are chosen such that they form an orthogonal family of functions in velocity space with respect to a weighted $L^{2}$-inner product that arises from entropy considerations for the linear Boltzmann equation [13]. The basis functions are constructed as products of polynomials and a Maxwellian; hence, they are most appropriate if the flow regime is not too far from a Maxwellian regime. This is suitable for the flows that will be considered here. Flows with other properties can however be approximated by this method by constructing different basis functions.

Inserting the expansion for $f^{(i)}(\mathbf{x}, \mathbf{v}, t)$ and testing successively against $\varphi_{k}(\mathbf{v})$ with respect to the inner product approximates (2.1) by a system of linear hyperbolic equations [13]

$$
\frac{\partial F^{(i)}}{\partial t}+A^{(1)} \frac{\partial F^{(i)}}{\partial x_{1}}+A^{(2)} \frac{\partial F^{(i)}}{\partial x_{2}}+A^{(3)} \frac{\partial F^{(i)}}{\partial x_{3}}=\frac{1}{\mathrm{Kn}} B^{(i)} F^{(i)}, \quad i=1, \ldots, n_{s},
$$

where $F^{(i)}(\mathbf{x}, t):=\left(f_{0}^{(i)}(\mathbf{x}, t), \ldots, f_{K-1}^{(i)}(\mathbf{x}, t)\right)^{T}$ is the vector of the $K$ coefficient functions in the expansion in velocity space. Here, $A^{(1)}, A^{(2)}, A^{(3)}$, and $B^{(i)}$ are constant $K \times K$ matrices. Using collocation basis functions, the coefficient matrices $A^{(1)}, A^{(2)}, A^{(3)}$ become diagonal matrices [4]. Note again that the equations for all species remain coupled through the crucial reaction boundary condition at the wafer surface.

The hyperbolic system (3.1) is now posed in a standard form as a system of partial differential equations on the spatial domain $\Omega \subset \mathbb{R}^{3}$ and in time $t$ and amenable to solution by various methods. Figure 1 shows two views of a representative domain $\Omega \subset \mathbb{R}^{3}$; more precisely, the plots show the solid wafer surface consisting of a 0.3 micrometer deep trench, in which is etched another 0.3 micrometer deep via (round hole). The domain $\Omega$ for our model is the gaseous region above the solid wafer surface up to the top of the plot box at $x_{3}=0.3$ micrometers in Figure 1. Since typical domains in our applications such as this one are of irregular shape, we use the discontinuous Galerkin method (DGM) [14], relying on a finite element discretization of the domain into tetrahedra.

The degrees of freedom (DOF) of the finite element method are the values of the $n_{s}$ species' coefficient functions $f_{\ell}^{(i)}(\mathbf{x}, t)$ in the Galerkin expansion at $K$ discrete velocities on the 4 vertices of each of the $N_{e}$ tetrahedra of the three-dimensional mesh. Hence, the complexity of the computational problem is given by $4 N_{e} K n_{s}$ at every time step. To appreciate the size of the problem, consider that the mesh of the domain in Figure 1 uses $N_{e}=7,087$ three-dimensional tetrahedral elements; even in the case of a single-species model $\left(n_{s}=1\right)$ and if we use just $K=4 \times 4 \times 4=64$ discrete velocities in three dimensions, as used for the application results in the following section, the total DOF are $N=1,814,272$ or nearly 2 million unknowns to be determined at every time step. Extensive validations of the numerical method and convergence studies 
for a wide range of Knudsen numbers are the focus of [4]. Based on these results, the choice of discrete velocities here is sufficient to obtain reliable results.

The size of problem at every time step motivates our interest in parallel computing. For the parallel computations on a distributed-memory cluster, the spatial domain $\Omega$ is partitioned in a pre-processing step, and the disjoint subdomains are distributed to separate parallel processes. The discontinuous Galerkin method for (3.1) needs the flux through the element faces. At the interface from one subdomain to the next, communications are required among those pairs of parallel processes that share a subdomain boundary. Additionally, a number of global reduce operations are needed to compute inner products, norms, and other diagnostic quantities. The performance of the parallel implementation was studied in [2] and confirmed that a distributed-memory cluster is very effective in speeding up calculations for a problem of this type.

\section{APPLICATION RESULTS}

As an application example, we present a model for chemical vapor deposition. In this process, reactants are supplied from the gas-phase interface at the top of the domain at $x_{3}=0.3$ in Figure 1 . The reactants flow downwards throughout the domain $\Omega$ until they reach the solid wafer surface shown in Figure 1, where some fraction of the molecules form a solid deposit. The time scale of all simulations corresponds to forming only a very thin layer; hence the surface is not moved within a simulation. Here, we use a single-species model with one reactive species $\left(n_{s}=1\right)$ and drop the species superscript in the following discussion. The deposition at the wafer surface can then be modeled using a sticking factor $0 \leq \gamma_{0} \leq 1$ that represents the fraction of molecules that are modeled to deposit at ("stick to") the wafer surface. The re-emission into $\Omega$ of gaseous molecules from the wafer surface is modeled as re-emission with velocity components in Maxwellian form and proportional to the flux to the surface as well as proportional to $1-\gamma_{0}$. The re-emission is scaled to conserve mass in the absence of deposition $\left(\gamma_{0}=0\right)$. The studies shown use a sticking factor of $\gamma_{0}=0.01$, that is, most molecules re-emit from the surface, which is a realistic condition [5]. The collision operator uses a relaxation time discretization by choosing $\sigma_{1}\left(\mathbf{v}, \mathbf{v}^{\prime}\right) \equiv 1 / \tau_{1}$ with (dimensionless) relaxation time $\tau_{1}=1.0$ that characterized the time to return to steady-state under appropriate boundary conditions. The temperature on the scale of this micron-scale domain is assumed constant and uniform throughout the domain and is set at $T=500 \mathrm{~K}[3]$. We focus on how the flow behaves when starting from no gas present throughout $\Omega$, modeled by initial condition $f \equiv 0$ at $t=0$ for the reactive species; the inert species is already present throughout the domain [3]. 


\subsection{Concentration Results}

Figures 2 through 5 show the results of transient simulations for the values of the Knudsen numbers $\mathrm{Kn}=0.01,0.1,1.0$, and 100.0, respectively, at six selected times throughout the simulation; notice that different times are selected for different cases. The quantity plotted for each (re-dimensionalized) time is the (dimensionless) concentration

$$
c(\mathbf{x}, t):=\int_{\mathbb{R}^{3}} f(\mathbf{x}, \mathbf{v}, t) d \mathbf{v}
$$

across the domain $\Omega$. The values of the dimensionless concentration $0 \leq c \leq 1$ is represented by the grayscale on each of the horizontal slices through $\Omega$ at the vertical levels at six values of $x_{3}$; the shapes of all slices together indicate the shape of the domain $\Omega$.

In Figure 2 for $\mathrm{Kn}=0.01$, a value that indicates near fluid-dynamic regime, we see that the top-most slice at $x_{3}=0.15$ is always darker than the slices below it, indicating higher concentration of molecules has reached this level from the inflow at the top of the domain than the lower levels deeper in the feature. More specifically, at the early times $5 \mathrm{~ns}$ and $10 \mathrm{~ns}$, relatively few molecules have reached the inside of the feature. By $10 \mathrm{~ns}$, the slice at $x_{3}=0$ shows that the concentration at the flat parts of the wafer surface has reached relatively high values than above the mouth of the trench $\left(0.3 \leq x_{1} \leq 0.7\right)$; this is explained by the ongoing flow of molecules into the trench. By $50 \mathrm{~ns}$, we observe the same phenomenon at the slice for $x_{3}=-0.3$, where the concentration has reached a higher value in the flat areas of the trench bottom as compared to the opening into the via (round hole) below. The following plots for times 100, 150, and 200 ns show how the fill of the entire domain with gaseous molecules continues over time.

Figure 3 shows slice plots of $c(\mathbf{x}, t)$ across $\Omega$ for the case of $\mathrm{Kn}=0.1$ at selected times $t$; notice that the first two plots are at the same times 5 and $10 \mathrm{~ns}$ as the previous figure, but the remaining times are different than in the previous figure. Comparing the plots at $t=5 \mathrm{~ns}$ in Figures 3 and 2 with each other, the one for the smaller Kn has generally lighter color indicating a slower fill of the feature with gas. The smaller Knudsen number means more collisions among molecules, leading to a less directional flow than for the larger Knudsen number. Since the bulk direction of the flow is downward because of the supply at the top with downward velocity, the feature fills faster with molecules in this case. This comparison also applies to time 10 ns. The following plots show again how the fill of the entire domain with gaseous molecules continues over time; as the times are 15, 20, 25, and 30 ns here, we see that the fill occurs substantially faster for this larger Knudsen number of $\mathrm{Kn}=0.1$.

Figures 4 and 5 extend the comparison of slice plots of $c(\mathbf{x}, t)$ to the Knudsen numbers $\mathrm{Kn}=1.0$ and 100.0, respectively; notice that smaller times are selected here, but the times 5 and $10 \mathrm{~ns}$ are still available for comparison, now in the final two plots. We notice first that the behavior of the two cases in Figures 4 
and 5 appears nearly identical, up to the resolution of the plots. We also note that the overall behavior of the feature filling with molecules is similar to the previous cases of smaller Knudsen numbers, but it clearly occurs a lot faster. By time $10 \mathrm{~ns}$, steady-state is rapidly being approached already, where in the previous plots at $10 \mathrm{~ns}$, in particular the inside of the via had only been reached by few molecules.

\subsection{Kinetic Density Results}

The previous figures showed results of the (macroscopic) concentration $c(\mathbf{x}, t)=\int f(\mathbf{x}, \mathbf{v}, t) d \mathbf{x}$, given as integral of the kinetic density $f(\mathbf{x}, \mathbf{v}, t)$. To provide more insight into the behavior of the solution, the following four figures show plots of the kinetic density $f(\mathbf{x}, \mathbf{v}, t)$ directly as a function of velocity $\mathbf{v}$. This requires fixing the spatial position $\mathrm{x} \in \Omega$ at which the kinetic density is analyzed. We select the values $\mathbf{x}=(0.5,0.5,0.0)$ at the center of the mouth of the trench (at height $\left.x_{3}=0.0\right)$ in Figures 6 and 7 and $\mathbf{x}=(0.5,0.5,-0.3)$ at the center of the mouth of the via (at height $\left.x_{3}=-0.3\right)$ in Figures 8 and 9 . We focus here on comparing the two Knudsen numbers $\mathrm{Kn}=0.01$ and 1.0, with the plots for $\mathrm{Kn}=0.01$ in Figures 6 and 8, and the plots for $\mathrm{Kn}=1.0$ in Figures 7 and 9. The selected times are, for each $\mathrm{Kn}$, the same ones as for the corresponding concentration plots in Figures 2 and 4, respectively.

Each plot in Figures 6 through 9 is an isosurface plot of $f(\mathbf{x}, \mathbf{v}, t)$ as function of $\mathbf{v} \in \mathbb{R}^{3}$, where the surface of the wireframe shown represents the isosurface level $f=0.005$. That is, the velocities on the inside of the wireframe have higher values of $f(\mathbf{x}, \mathbf{v}, t)$ and the outside lower values than 0.005 . This presentation is chosen, because a Maxwellian distribution of the molecule velocities, that represents the case of fully randomized velocities, is useful as a reference and would result in a sphere in three dimensions, up to the resolution of the velocity discretization.

In Figure 6 for the velocity distribution at the mouth of the trench at $\mathbf{x}=(0.5,0.5,0.0)$ for the relatively small Knudsen number $\mathrm{Kn}=0.01$, the plots at times 5 and $10 \mathrm{~ns}$ confirm that few molecules have reached this point, as all components of $f(\mathbf{x}, \mathbf{v}, t)$ are less than 0.005. In accordance with the concentration results in Figure 2, by time 50 ns, significant amounts of molecules have reached the mouth of the trench. The plots in Figure 6 for the times 50 ns and larger additionally show that these molecules have apparently a randomized velocity distribution, close to a Maxwellian distribution, for $\mathrm{Kn}=0.01$.

By contrast, the plots in Figure 7 for the case of the larger $\mathrm{Kn}=1.0$ first of all confirm the results in Figure 4 that molecules reach the position $\mathbf{x}=(0.5,0.5,0.0)$ faster; notice that the times 5 and $10 \mathrm{~ns}$ are in the bottom row of plots in this figure. But we also see in the plots for the earlier times 2, 3, $4 \mathrm{ns,}$ and even at $5 \mathrm{~ns}$, that the distribution of velocities is far from Maxwellian. Rather, as $f(\mathbf{x}, \mathbf{v}, t)>0.005$ only for $v_{3}<0$, we have predominantly velocities pointing from the inlet at the top of the domain downward into the feature. By 10 ns though, sufficiently many molecules have been re-emitted from the wafer surface that 
there are also many molecules with upward velocities.

The next two Figures 8 and 9 show the same quantity as the previous plots at the same times, but with the point $\mathbf{x}=(0.5,0.5,-0.3)$ chosen at the mouth of the via (round hole) half-way down the feature. Since this position lies deeper inside the feature than $\mathbf{x}=(0.5,0.5,0.0)$, it is expected that there is a time lag for molecules to reach this position. This can be seen for each Kn by comparing Figure 8 with Figure 6 for $\mathrm{Kn}=0.01$ and Figure 9 with Figure 7 for $\mathrm{Kn}=1.0$. Particularly, the kinetic density at $t=50 \mathrm{~ns}$ in Figure 8 has smaller values than those in Figure 6, but they are equally randomized due to relatively frequent collisions for $\mathrm{Kn}=0.01$. Analogously, the density at $t=5 \mathrm{~ns}$ in Figure 9 has smaller values than the density in Figure 7, but it is also still more directional and less randomized.

\subsection{Kinetic Saturation Results}

The final plots in Figures 6 through 9 all appear to show that kinetic density has reached a Maxwellian velocity distribution in the final plot. To determine if this is indeed the case, we now plot the kinetic saturation $0 \leq f(\mathbf{x}, \mathbf{v}, t) / M(\mathbf{v}) \leq 1$ that shows how close to a Maxwellian the kinetic density $f$ is. Moreover, all results in the previous four figures bear out that at positions $\mathbf{x}$ in the center of the feature $\left(x_{1}=x_{2}=0.5\right)$ the kinetic density $f(\mathbf{x}, \mathbf{v}, t)$ does not depend on the velocity components $v_{1}$ and $v_{2}$ in the $x_{1}$ - and $x_{2}$-directions, respectively, and we use this observation to plot $f / M$ as a function of $v_{3}$ only, for $v_{1}=v_{2}=0$ fixed, in Figures 10 and 11. Each line shows the result at a particular time, as listed in the figure captions; these times are the same ones for each Kn as before, but different for the two Kn.

Figure 10 shows the saturation $f / M$ at the trench mouth and via mouth for $\mathrm{Kn}=0.01$. In both cases, the saturation increases over time. Overall, the saturation levels are lower at the via mouth than at the trench mouth, which is consistent with the concentration results in Figure 2; notice the different scales on the vertical axes. We notice that the last lines are closer to each other than earlier lines, so a steady-state is being approached; notice that only the final four times are uniformly spaced. Finally, each line shows larger values of $f / M$ for $v_{3}<0$ (the left side of the plot) than for $f_{3}>0$ (the right side). This means that the velocity distribution has a downward direction. This detail could not be clearly determined in Figure 6 or 8 ; in fact, many of the plots of the kinetic density could not be distinguished from Maxwellians there, but the present plots of the saturation allow us to establish clearly that they are not Maxwellians.

Figure 11 shows the saturation $f / M$ for $\mathrm{Kn}=1.0$. The same observations as for Figure 10 hold concerning the level of saturation being higher at the trench mouth than the via mouth and concerning a generally downward velocity distribution. But additionally, we notice that the difference between the $f / M$ values for $v_{3}<0$ and $v_{3}>0$ is sharper for most of the times; this corresponds to fewer collisions for this larger $\mathrm{Kn}=1.0$ compared to more collisions for $\mathrm{Kn}=0.01$ that tend to smooth out the velocity distribution. However, by 
the final time, sufficiently many molecules have been re-emitted from the wafer surface that also upward velocity components are present in the distribution and hence the saturation plot has become more uniform.

\section{CONCLUSIONS}

The results presented in the previous section highlight the capabilities of the KTRM and the numerical method used to model an important chemical process. We see that the flow associated with larger Knudsen numbers is more directional and the transients due to the change in flux of reactant species are shorter for higher Kn. This is highlighted by looking at the local kinetic density function at selected times. Access to the kinetic density is thus important to understanding such systems.

The studies presented point out the need to consider Kn when deciding how long transients due to perturbations might last. That is, when deciding the simulation time for a particular case, its Knudsen number must be taken into account, because different times will be appropriate for different Kn. This is important in our effort to develop a multiscale simulator for processes of this type.

\section{ACKNOWLEDGMENTS}

The hardware used in the computational studies was partially supported by the SCREMS grant DMS0215373 from the U.S. National Science Foundation with additional support from the University of Maryland, Baltimore County. See www.math.umbc.edu/ gobbert/kali for more information on the machine and the projects using it. Prof. Cale acknowledges support from MARCO, DARPA, and NYSTAR through the Interconnect Focus Center. We also thank Max O. Bloomfield for supplying the original mesh of the domain.

\section{REFERENCES}

[1] Kersch, A., Morokoff, W.J.: Transport Simulation in Microelectronics. Volume 3 of Progress in Numerical Simulation for Microelectronics. Birkhäuser Verlag, Basel (1995)

[2] Gobbert, M.K., Breitenbach, M.L., Cale, T.S.: Cluster computing for transient simulations of the linear Boltzmann equation on irregular three-dimensional domains. In Sunderam, V.S., van Albada, G.D., Sloot, P.M.A., Dongarra, J.J., eds.: Computational Science-ICCS 2005. Volume 3516 of Lecture Notes in Computer Science., Springer-Verlag (2005) 41-48

[3] Gobbert, M.K., Cale, T.S.: A kinetic transport and reaction model and simulator for rarefied gas flow in the transition regime. J. Comput. Phys., in press (2006) 
[4] Gobbert, M.K., Webster, S.G., Cale, T.S.: A Galerkin method for the simulation of the transient 2-D/2-D and 3-D/3-D linear Boltzmann equation. J. Sci. Comput., accepted (2006)

[5] Cale, T.S., Merchant, T.P., Borucki, L.J., Labun, A.H.: Topography simulation for the virtual wafer fab. Thin Solid Films 365 (2000) 152-175

[6] Gobbert, M.K., Merchant, T.P., Borucki, L.J., Cale, T.S.: A multiscale simulator for low pressure chemical vapor deposition. J. Electrochem. Soc. 144 (1997) 3945-3951

[7] Merchant, T.P., Gobbert, M.K., Cale, T.S., Borucki, L.J.: Multiple scale integrated modeling of deposition processes. Thin Solid Films 365 (2000) 368-375

[8] Gobbert, M.K., Cale, T.S.: A feature scale transport and reaction model for atomic layer deposition. In Swihart, M.T., Allendorf, M.D., Meyyappan, M., eds.: Fundamental Gas-Phase and Surface Chemistry of Vapor-Phase Deposition II. Volume 2001-13., The Electrochemical Society Proceedings Series (2001) $316-323$

[9] Gobbert, M.K., Webster, S.G., Cale, T.S.: Transient adsorption and desorption in micrometer scale features. J. Electrochem. Soc. 149 (2002) G461-G473

[10] Gobbert, M.K., Prasad, V., Cale, T.S.: Modeling and simulation of atomic layer deposition at the feature scale. J. Vac. Sci. Technol. B 20 (2002) 1031-1043

[11] Gobbert, M.K., Prasad, V., Cale, T.S.: Predictive modeling of atomic layer deposition on the feature scale. Thin Solid Films 410 (2002) 129-141

[12] Prasad, V., Gobbert, M.K., Bloomfield, M., Cale, T.S.: Improving pulse protocols in atomic layer deposition. In Melnick, B.M., Cale, T.S., Zaima, S., Ohta, T., eds.: Advanced Metallization Conference 2002, Materials Research Society (2003) 709-715

[13] Ringhofer, C., Schmeiser, C., Zwirchmayr, A.: Moment methods for the semiconductor Boltzmann equation on bounded position domains. SIAM J. Numer. Anal. 39 (2001) 1078-1095

[14] Remacle, J.F., Flaherty, J.E., Shephard, M.S.: An adaptive discontinuous Galerkin technique with an orthogonal basis applied to compressible flow problems. SIAM Rev. 45 (2003) 53-72 

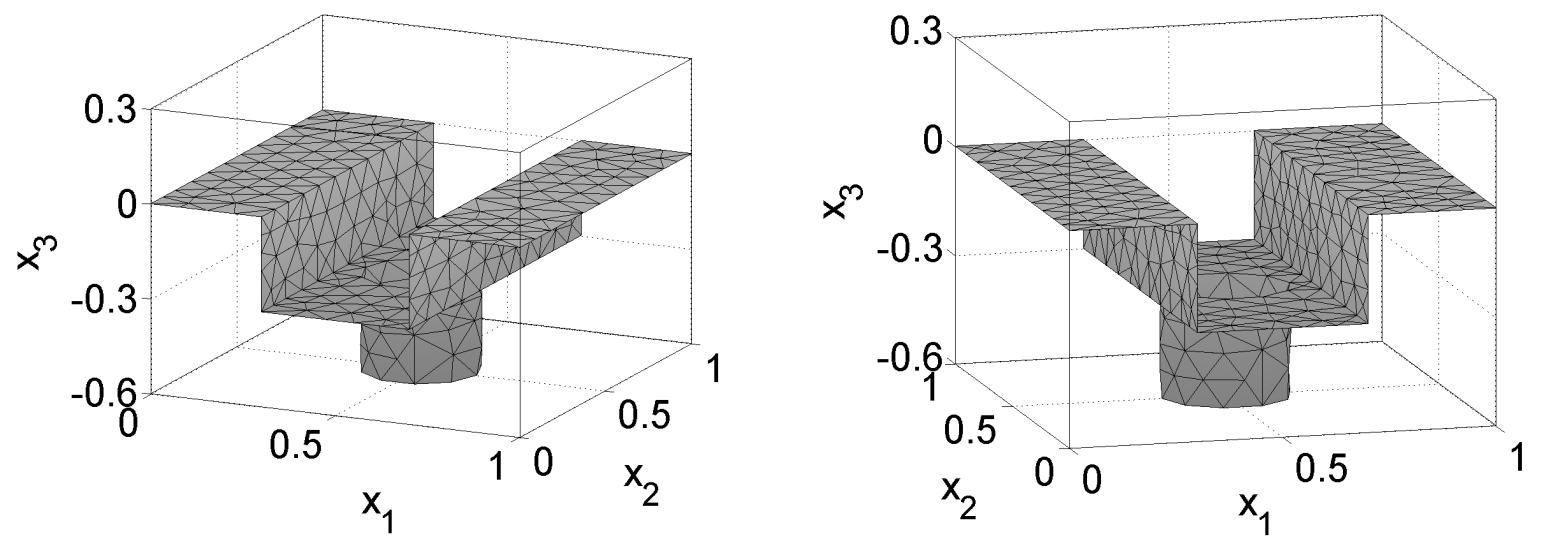

Figure 1: Two views of the solid wafer surface boundary of the trench/via domain. The gas domain of the model is the region above the wafer surface up to the top of the plot box. 

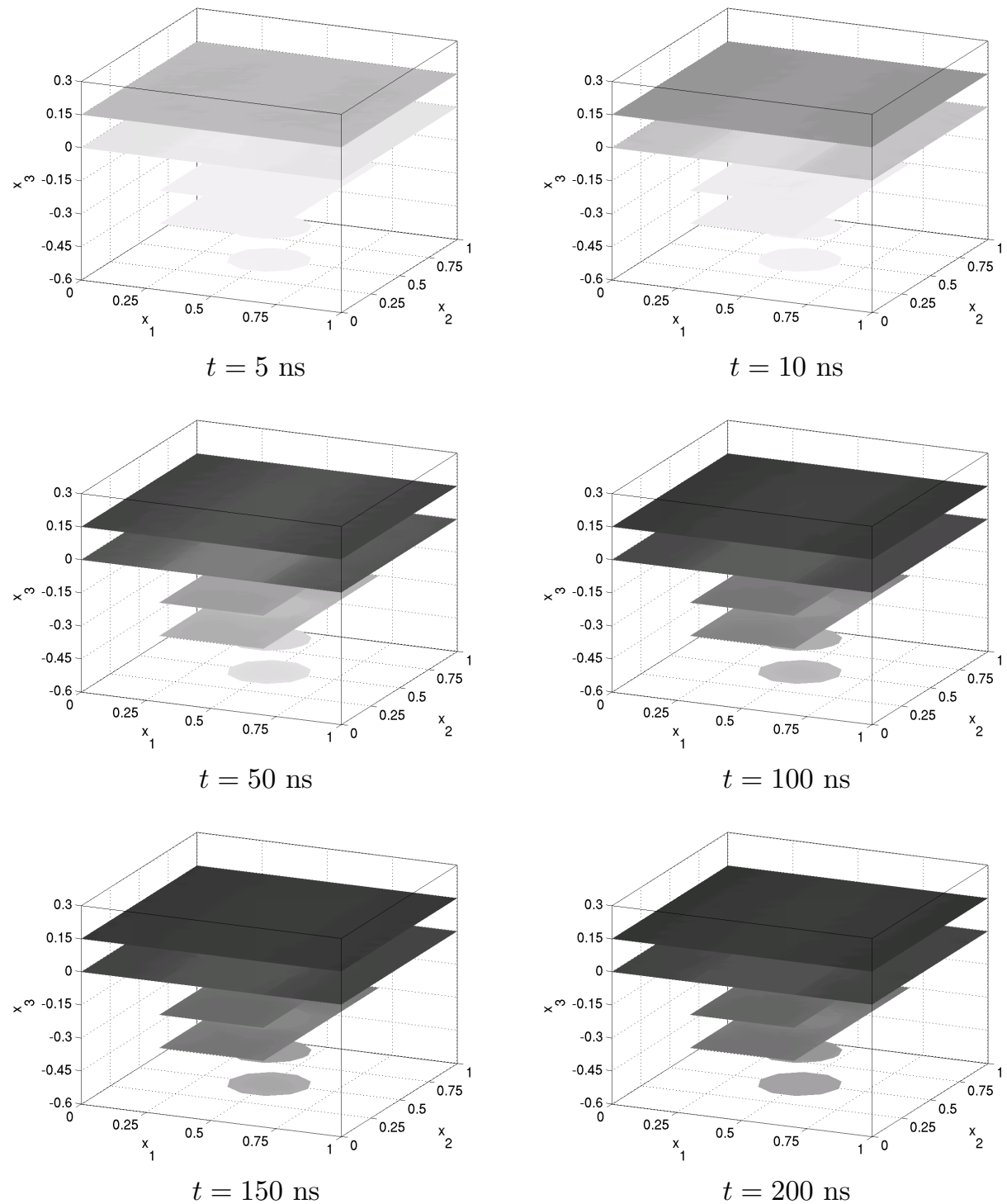

Figure 2: Slice plots of the dimensionless concentration $c(\mathbf{x}, t)$ for $\mathrm{Kn}=0.01$ at heights $x_{3}=$ $-0.60,-0.45,-0.30,-0.15,0.00,0.15$ at selected times $t$. Grayscale from light $\Leftrightarrow c=0$ to dark $\Leftrightarrow c=1$. 

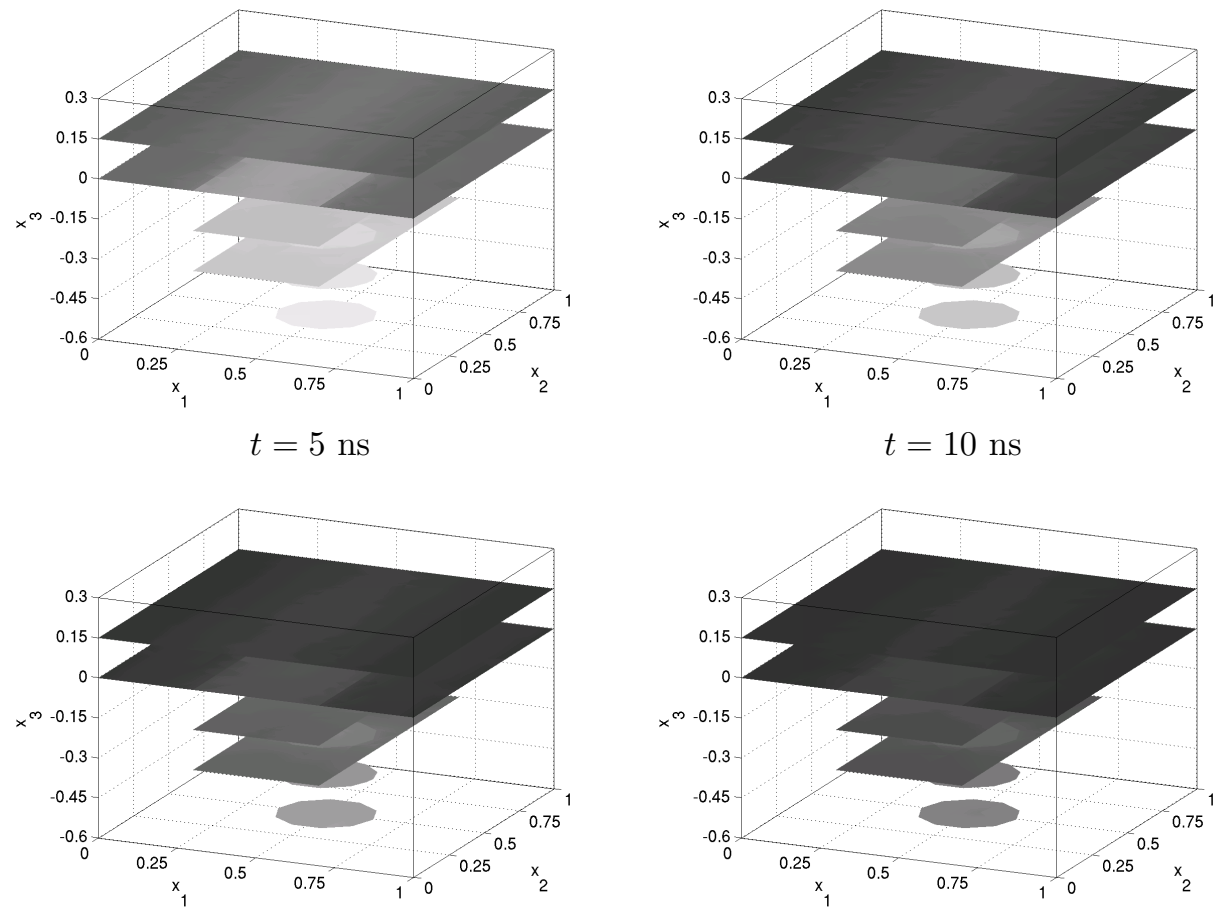

$$
t=15 \mathrm{~ns}
$$

$$
t=20 \mathrm{~ns}
$$
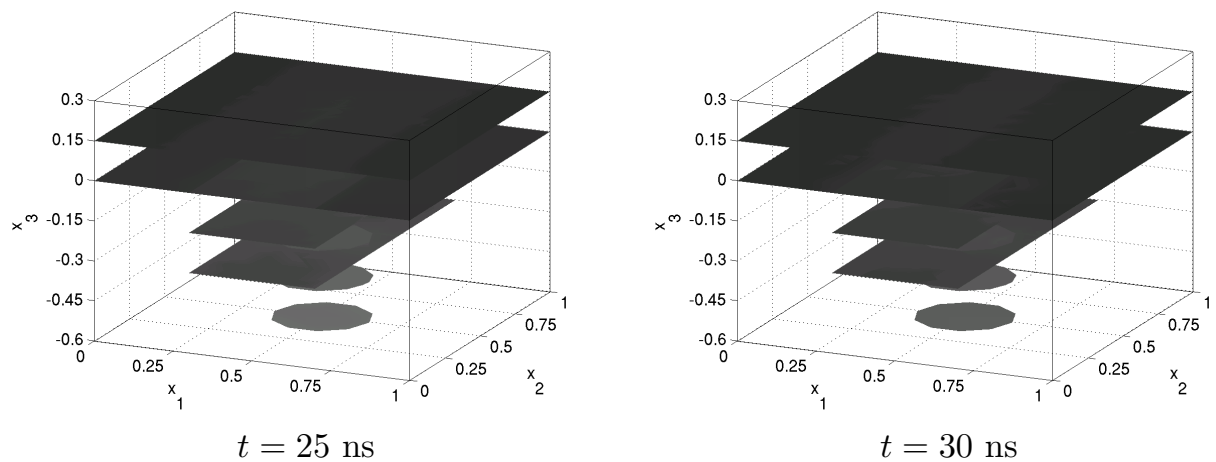

Figure 3: Slice plots of the dimensionless concentration $c(\mathbf{x}, t)$ for $\mathrm{Kn}=0.1$ at heights $x_{3}=$ $-0.60,-0.45,-0.30,-0.15,0.00,0.15$ at different times $t$. Grayscale from light $\Leftrightarrow c=0$ to dark $\Leftrightarrow c=1$. 

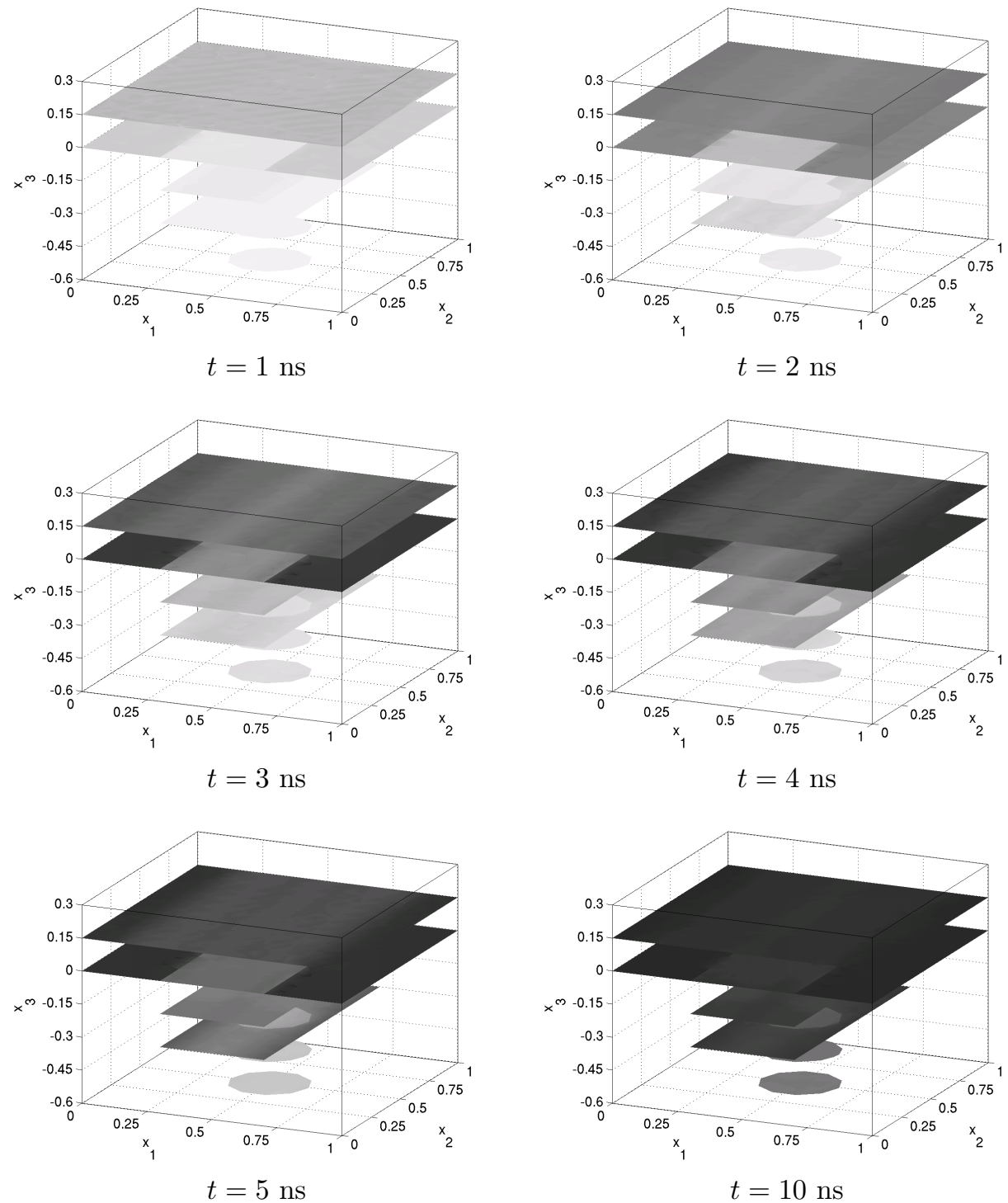

Figure 4: Slice plots of the dimensionless concentration $c(\mathbf{x}, t)$ for $\mathrm{Kn}=1.0$ at heights $x_{3}=$ $-0.60,-0.45,-0.30,-0.15,0.00,0.15$ at different times $t$. Grayscale from light $\Leftrightarrow c=0$ to dark $\Leftrightarrow c=1$. 

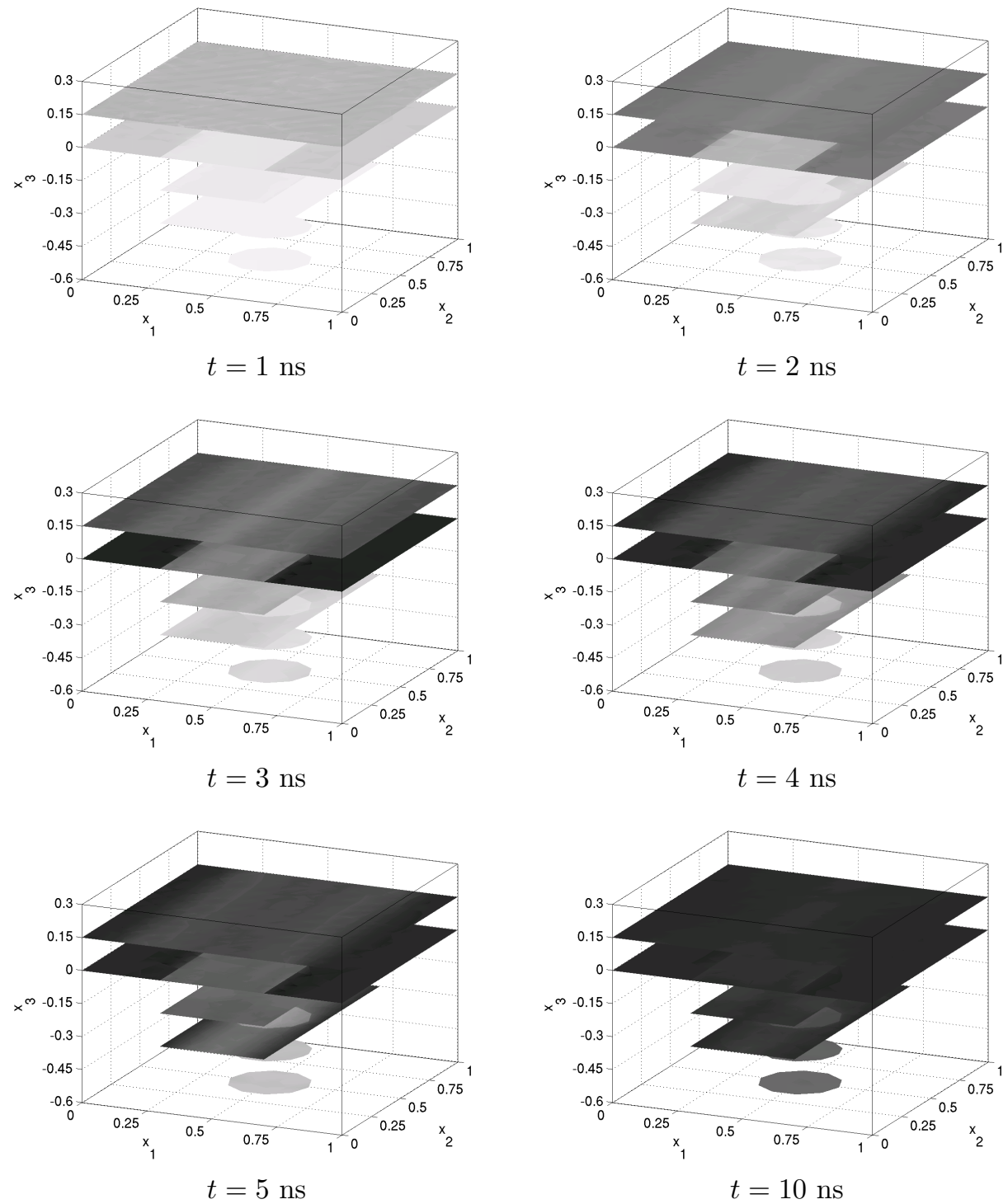

Figure 5: Slice plots of the dimensionless concentration $c(\mathrm{x}, t)$ for $\mathrm{Kn}=100.0$ at heights $x_{3}=$ $-0.60,-0.45,-0.30,-0.15,0.00,0.15$ at different times $t$. Grayscale from light $\Leftrightarrow c=0$ to dark $\Leftrightarrow c=1$. 

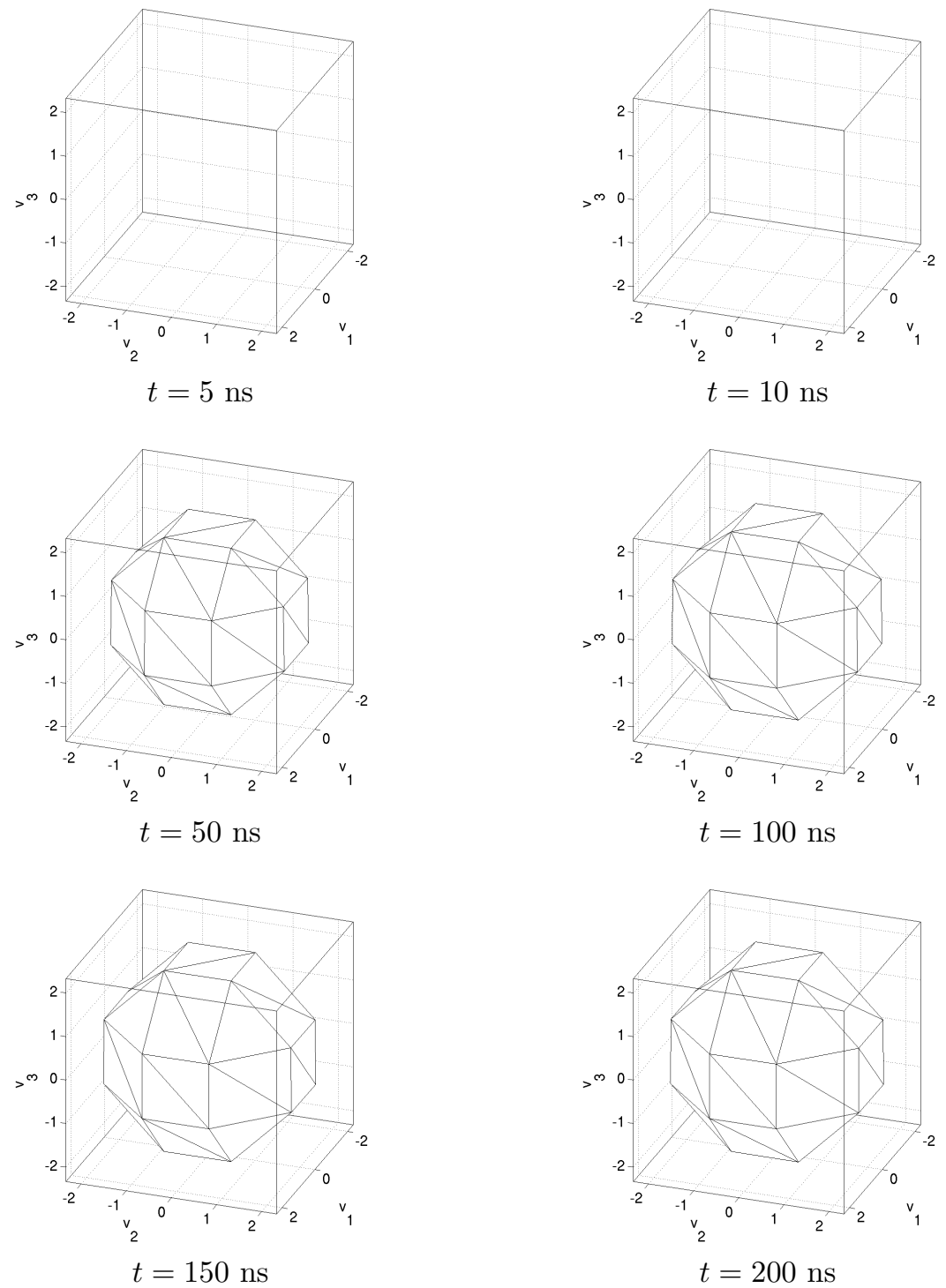

Figure 6: Isosurface plots of the kinetic density $f(\mathbf{x}, \mathbf{v}, t)$ for $\mathrm{Kn}=0.01$ as function of velocity $\mathbf{v} \in \mathbb{R}^{3}$ at the mouth of the trench at $\mathbf{x}=(0.5,0.5,0.0)$ at selected times. Isosurface level at $f(\mathbf{x}, \mathbf{v}, t)=0.005$. 

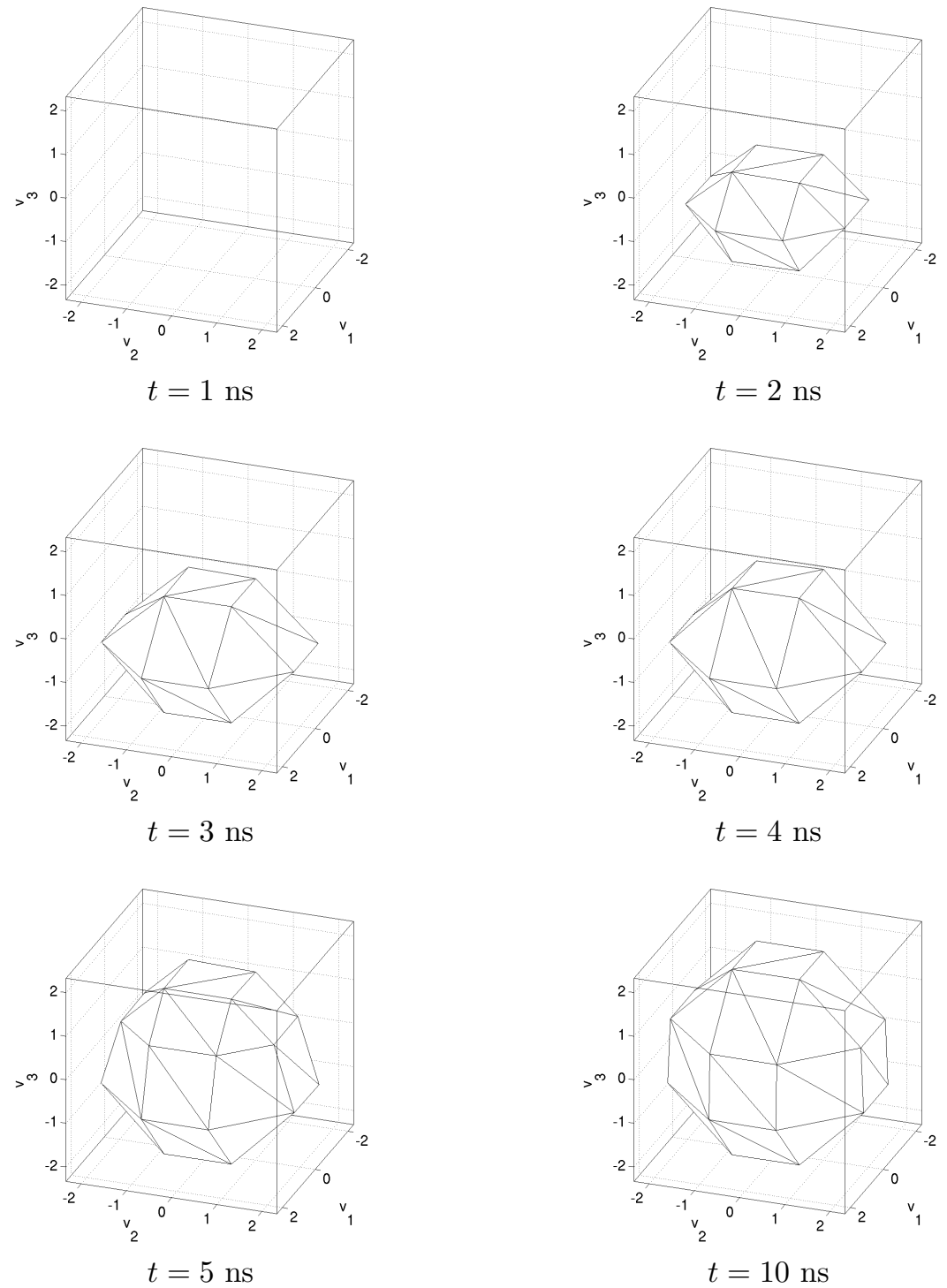

Figure 7: Isosurface plots of the kinetic density $f(\mathbf{x}, \mathbf{v}, t)$ for $\mathrm{Kn}=1.0$ as function of velocity $\mathbf{v} \in \mathbb{R}^{3}$ at the mouth of the trench at $\mathbf{x}=(0.5,0.5,0.0)$ at selected times. Isosurface level at $f(\mathbf{x}, \mathbf{v}, t)=0.005$. 

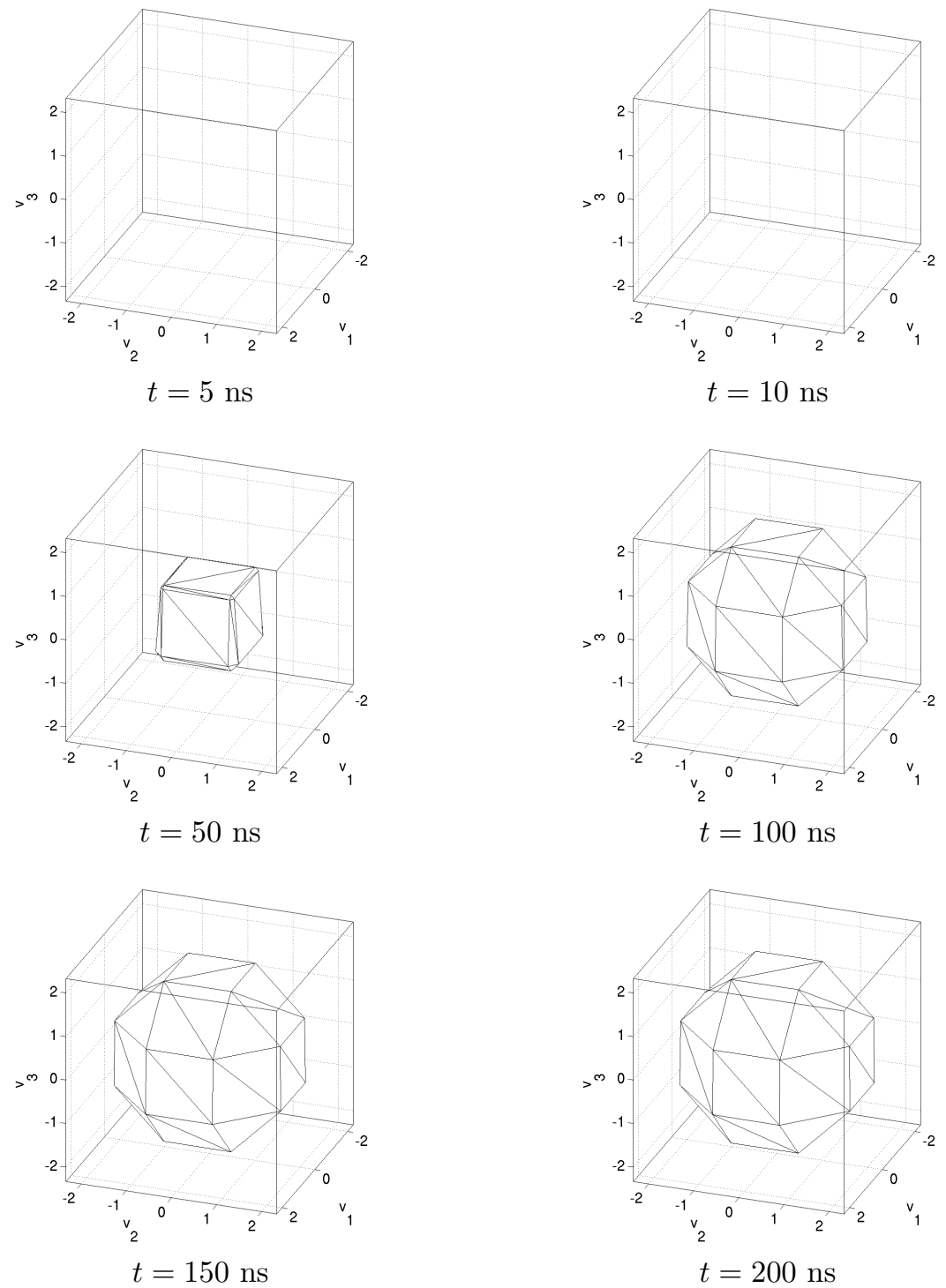

Figure 8: Isosurface plots of the kinetic density $f(\mathbf{x}, \mathbf{v}, t)$ for $\mathrm{Kn}=0.01$ as function of velocity $\mathbf{v} \in \mathbb{R}^{3}$ at the mouth of the via at $\mathbf{x}=(0.5,0.5,-0.3)$ at selected times. Isosurface level at $f(\mathbf{x}, \mathbf{v}, t)=0.005$. 

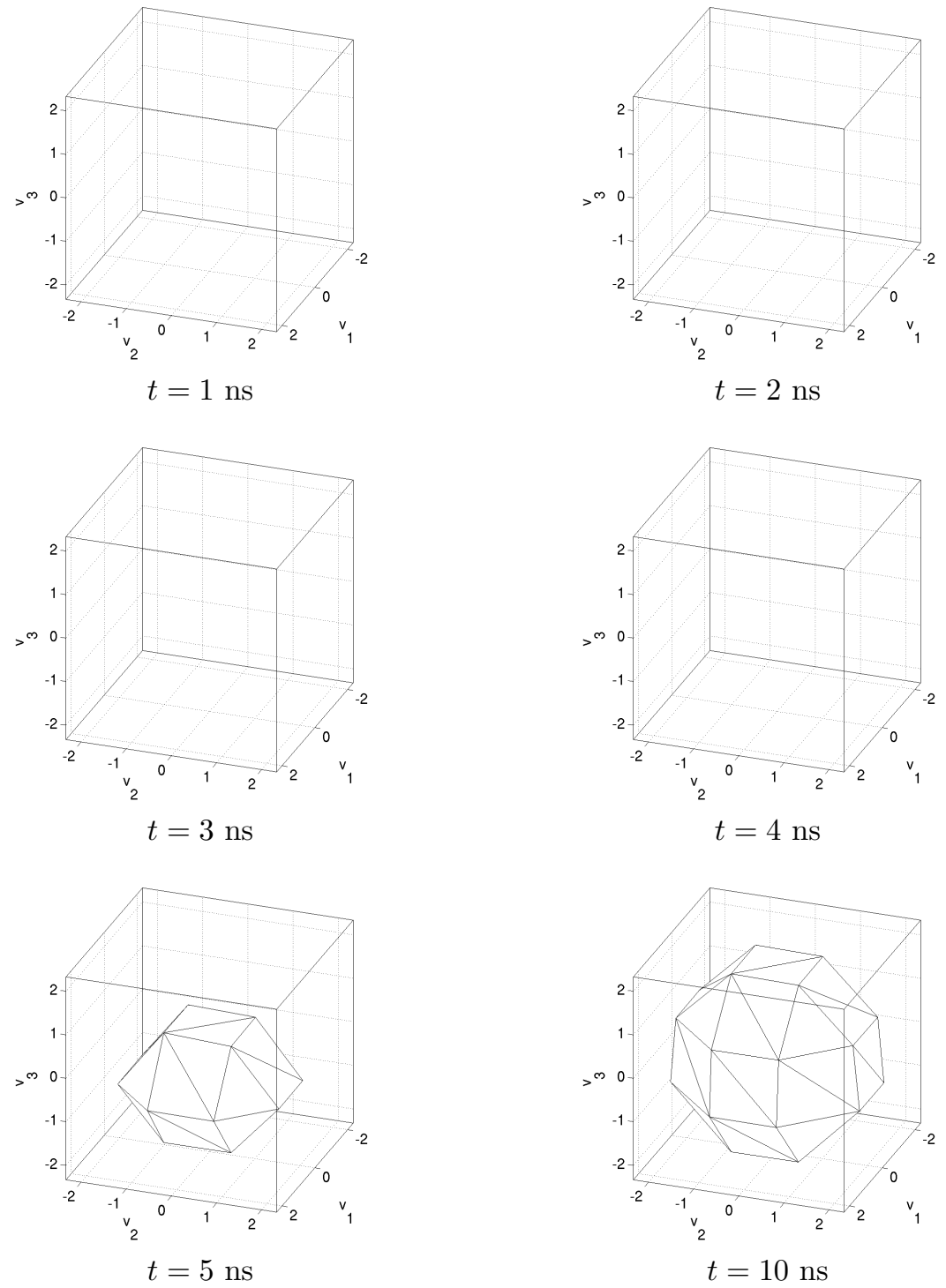

Figure 9: Isosurface plots of the kinetic density $f(\mathbf{x}, \mathbf{v}, t)$ for $\mathrm{Kn}=1.0$ as function of velocity $\mathbf{v} \in \mathbb{R}^{3}$ at the mouth of the via at $\mathbf{x}=(0.5,0.5,-0.3)$ at selected times. Isosurface level at $f(\mathbf{x}, \mathbf{v}, t)=0.005$. 

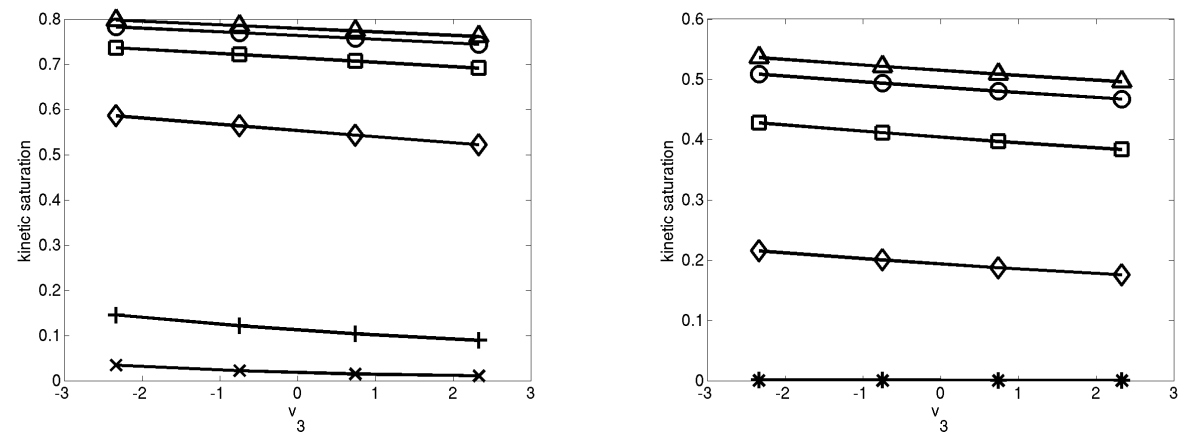

trench mouth

via mouth

Figure 10: Line plots of the saturation of the kinetic density $f(\mathbf{x}, \mathbf{v}, t) / M(\mathbf{v})$ for $\operatorname{Kn}=0.01$ for $\left(v_{1}, v_{2}\right)=(0,0)$ as function of $v_{3}$; at the mouth of the trench at position $\mathbf{x}=(0.5,0.5,0.0)$ and at the mouth of the via at position $\mathbf{x}=(0.5,0.5,-0.3)$; at times: $\times=5 \mathrm{~ns},+=10 \mathrm{~ns}, \diamond=50 \mathrm{~ns}, \square=100 \mathrm{~ns}, \circ=150 \mathrm{~ns}, \triangle=200 \mathrm{~ns}$. Notice the different scales of the vertical axes.

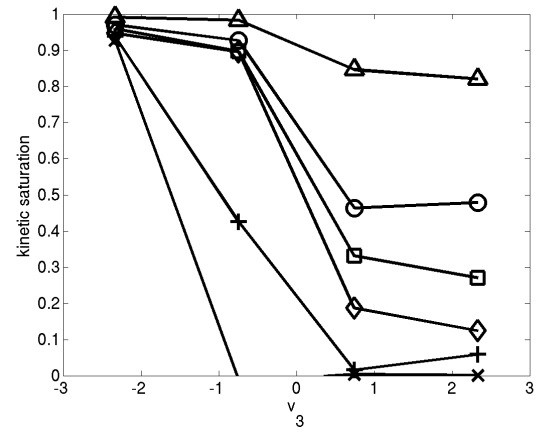

trench mouth

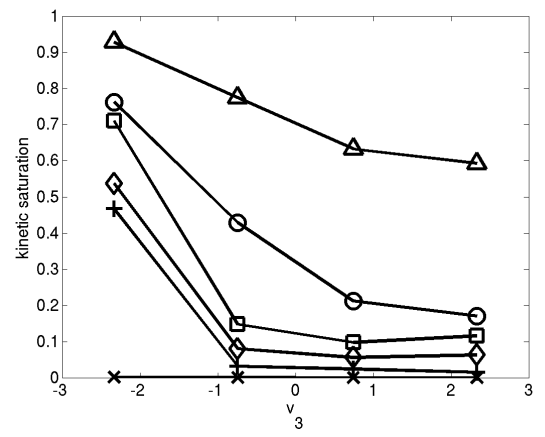

via mouth

Figure 11: Line plots of the saturation of the kinetic density $f(\mathbf{x}, \mathbf{v}, t) / M(\mathbf{v})$ for $\mathrm{Kn}=1.0$ for $\left(v_{1}, v_{2}\right)=(0,0)$ as function of $v_{3}$; at the mouth of the trench at position $\mathbf{x}=(0.5,0.5,0.0)$ and at the mouth of the via at position $\mathbf{x}=(0.5,0.5,-0.3)$; at times: $\times=1 \mathrm{~ns},+=2 \mathrm{~ns}, \diamond=3 \mathrm{~ns}, \square=4 \mathrm{~ns}, \circ=5 \mathrm{~ns}, \triangle=10 \mathrm{~ns}$. 